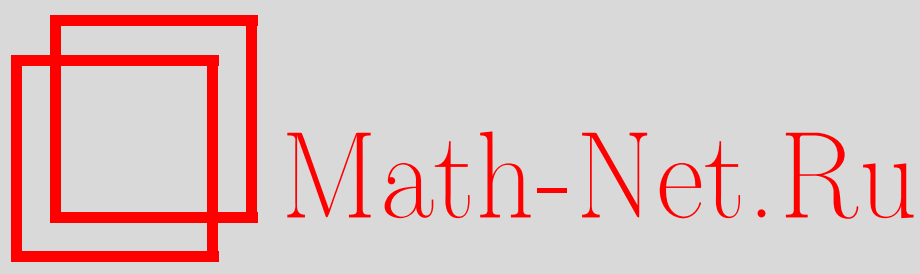

С. Г. Мерзляков, О замкнутости образа разностного оператора, Изв. РАН. Сер. матем., 1997, том 61, выпуск 3, 133-158

DOI: https://doi.org/10.4213/im128

Использование Общероссийского математического портала Math-Net.Ru подразумевает, что вы прочитали и согласны с пользовательским соглашением

http://www . mathnet.ru/rus/agreement

Параметры загрузки:

IP: 54.84 .234 .179

26 апреля 2023 г., 14:35:52 
УДК 517.5

\section{С.Г. Мерзляков}

\section{О замкнутости образа разностного оператора}

В статье исследуются разностные операторы с переменными разностями, лежащими на одной прямой, в пространствах голоморфных функций одной переменной. Получены необходимые и достаточные условия замкнутости образа таких операторов в терминах нулей его крайних коэффициентов. Для широкого класса областей эти условия дают простой критерий замкнутости образа разностного оператора.

Библиография: 7 наименований.

\section{Введение}

Для произвольной области $G$ комплексной плоскости через $H(G)$ будем обозначать пространство голоморфных функций в этой области с топологией равномерной сходимости на компактах.

Пусть область $U \subset \mathbb{C}$ и числа $0=s_{0}<s_{1}<\cdots<s_{r}<\infty$ таковы, что множество

$$
D:=\bigcap_{j=0}^{r}\left(U-i s_{j}\right)
$$

непусто и связно. Через $i$ всюду в дальнейшем будет обозначаться мнимая единица.

Для заданных функций $g_{0}, g_{1}, \ldots, g_{r} \in H(D)$,

$$
g_{0} \not \equiv 0 \not \equiv g_{r},
$$

рассмотрим разностный оператор из пространства $H(U)$ в $H(D)$, действующий по формуле

$$
(\mathscr{A} f)(z)=\sum_{j=0}^{r} g_{j}(z) f\left(z+i s_{j}\right), \quad f \in H(U) .
$$

Ясно, что это - линейный непрерывный оператор.

В данной статье будут получены необходимые и достаточные условия замкнутости образа оператора $\mathscr{A}$ в терминах нулей его крайних коэффициентов $-g_{0}$ и $g_{r}$. Для широкого класса областей эти условия дадут простой критерий замкнутости образа оператора $\mathscr{A}$.

Мы будем предполагать, что функции $g_{0}, \ldots, g_{r}$ не имеют общих нулей. Как несложно показать, это условие не ограничивает обшности.

Результаты статьи были анонсированы в работе автора [1].

Работа выполнена при финансовой поддержке РФФИ (грант 93-011-259).

$$
\text { (C) С.Г. Мерзляков } 1997
$$




\section{§ 1. Предварительные результаты}

Для односвязной области $G$ через $H^{*}(G)$ обозначим сильное сопряженное пространство к пространству $H(G)$.

Как известно, каждому функционалу $S$ из пространства $H^{*}(G)$ однозначно соответствует такая голоморфная вне некоторого компакта $K \subset G$ функция $\gamma(z)$, $\gamma(\infty)=0$, что для любого замкнутого контура $C$ в области $G$, охватываюшего компакт $K$, и любой функции $f \in H(G)$ имеем

$$
\langle S, f\rangle=\frac{1}{2 \pi i} \int_{C} \gamma(t) f(t) d t
$$

(см. [2]). Функцию $\gamma$ назовем преобразованием Коши функционала $S$.

Эта функция $\gamma(z)$ будет голоморфна в неограниченной связной компоненте дополнения некоторого компакта $K_{1} \subset G$ тогда и только тогда, когда для любого числа $\varepsilon>0$ найдется число $c>0$ такое, что для любой функции $f \in H(G)$ выполнено неравенство

$$
|\langle S, f\rangle| \leqslant c \max _{z \in K_{1}^{\varepsilon}}|f(z)|,
$$

где $K_{1}^{\varepsilon}-\varepsilon$-вздутие компакта $K_{1}$ (см. [2]). Такой компакт $K_{1}$ называется определяющим множеством функционала $S$.

Рассмотрим оператор $\mathscr{A}^{*}$, сопряженный к оператору $\mathscr{A}$, отображающий пространство $H^{*}(D)$ в $H^{*}(U)$.

Из сказанного вьше ясно, что если функция $\gamma(z)$, являющаяся преобразованием Коши функционала $S \in H^{*}(D)$, голоморфна вне компакта $k \subset D$, то преобразование Коши $\eta(z)$ функционала $\mathscr{A}^{*} S$ будет голоморфно в неограниченной связной компоненте дополнения компакта

$$
\bigcup_{j=0}^{r}\left(k+i s_{j}\right)
$$

Для изучения обратной связи между функциями $\eta(z)$ и $\gamma(z)$ нам понадобится следующий результат.

ЛЕмма 1. В окрестности бесконечности имеет место представление

$$
\eta(z)=\sum_{j=0}^{r} \frac{1}{2 \pi i} \int_{C} \frac{\gamma(t) g_{j}(t)}{z-t-i s_{j}} d t
$$

где контур $C \subset D$ охватывает все особенности функиии $\gamma(z)$.

ДокАЗАТЕЛЬСТво. Пусть контур $\Gamma \subset U$ охватывает все особенности функции $\eta(z)$. Из определения сопряженного оператора получим

$$
\frac{1}{2 \pi i} \int_{\Gamma} \eta(t) f(t) d t=\frac{1}{2 \pi i} \int_{C} \gamma(t)\left[\sum_{j=0}^{r} g_{j}(t) f\left(t+i s_{j}\right)\right] d t, \quad f \in H(U) .
$$

Если точка $z$ принадлежит неограниченной связной компоненте дополнения компакта

$$
\Gamma \cup \bigcup_{j=0}^{r}\left(C+i s_{j}\right)
$$


то ядро Коши $(z-t)^{-1}$ можно сколь угодно точно аппроксимировать по переменной $t$ на этом компакте функциями пространства $H(U)$.

Для таких точек $z$ из последнего равенства по непрерывности следует

$$
\eta(z)=\frac{1}{2 \pi i} \int_{\Gamma} \frac{\eta(z)}{z-t} d t=\sum_{j=0}^{r} \frac{1}{2 \pi i} \int_{C} \frac{\gamma(t) g_{j}(t)}{z-t-i s_{j}} d t
$$

что и требовалось доказать.

ОПРЕДЕЛЕНИЕ 1 . Назовем множество $M \subset \mathbb{C} i$-вылуклым, если его пересечение с любой прямой, параллельной мнимой оси, связно.

Как несложно показать, $i$-выпуклые области будут обязательно односвязными, а $i$-выпуклые компакты имеют связное дополнение.

Отныне будем считать нашу область $U i$-выпуклой. В таком случае и область $D$, очевидно, также $i$-выпукла.

ЛЕмма 2. Пусть $S \in H^{*}(D)$, функиии $\gamma(z)$ и $\eta(z)$ являются преобразованиями Коши соответственно функиионалов $S$ и $\mathscr{A}^{*} S$. Пусть, далее, функция $\eta(z)$ голоморфна на лучах

$$
\left\{h_{0}+i y: y<0\right\}, \quad\left\{h_{1}+i y: y>0\right\}
$$

для некоторых точек $h_{0}, h_{1} \in \mathbb{C}$ таких, что $h_{0},\left(h_{1}-i s_{r}\right) \in D$.

Тогда функиия $\gamma$ будет мероморфно продолжсться из бесконечности по лучам

$$
\left\{h_{0}+i y: y<0\right\}, \quad\left\{h_{1}+i y-i s_{r}: y>0\right\},
$$

причем полюсов на этих лучах будет конечное множество и самые дальние от начал лучей полюса будут нулями соответственно функиий $g_{0}(z)$ и $g_{r}(z)$.

ДокАЗАТЕЛЬСТво. Ограничимся случаем луча, уходящего вниз.

Функция $\gamma(z)$ голоморфна вне некоторого компакта области $D$, следовательно, найдется число $a \in \mathbb{R}$ такое, что на луче $\left\{h_{0}+i y+i a: y<0\right\}$ у этой функции нет особенностей.

Если число $a$ неотрищательно, то доказывать нечего, поэтому предположим, что $a<0$ и точка $\left(h_{0}+i a\right)$ особая для функции $\gamma(z)$, и обозначим через $b$ точную верхнюю грань чисел $c \leqslant 0$, для которых на луче

$$
\left\{h_{0}+i y: y<c\right\}
$$

у функции $\gamma(z)$ нет других особенностей, кроме полюсов.

Как показано в статье автора [3, с. 1247], в области $D$ существует кусочно гладкий контур $C$, охватьвающий все особенности функции $\gamma$ и пересекающийся с любой вертикальной прямой не более чем в двух точках. Деформируя контур $C$ в представлении (2), найдем, что все слагаемые в правой части этого равенства, начиная со второго, будут голоморфны на луче

$$
\left\{h_{0}+i y: y<a+s_{1}\right\}
$$

а на луче

$$
\left\{h_{0}+i y: y<b+\varepsilon\right\}
$$


где $0<\varepsilon<s_{1}$, имеют конечное число полюсов.

В таком случае и первое слагаемое будет голоморфино на луче

$$
\left\{h_{0}+i y: y<\min \left(a+s_{1}, 0\right)\right\} \text {, }
$$

а на луче

$$
\left\{h_{0}+i y: y<\min (b+\varepsilon, 0)\right\}
$$

имеет ограниченное по $\varepsilon$ число полюсов. Из формулы Сохоцкого-Племеля заключаем, что этим же свойством будет обладать и функция $\gamma(z) g_{0}(z)$.

Следовательно, в точке $\left(h_{0}+i a\right)$ у функции $\gamma(z)$ будет полюс, у функции $g_{0}(z)-$ корень, а на луче

$$
\left\{h_{0}+i y: y<\min (b+\varepsilon, 0)\right\}
$$

у функции $\gamma(z)$ конечное число полюсов, ибо на отрезке $\left[h_{0}, h_{0}+i a\right]$ функция $g_{0}(z)$ голоморфна. Из определения числа $b$ и произвольности числа $\varepsilon, 0<\varepsilon<s_{1}$, заключаем, что $b=0$. Лемма полностью доказана.

Для множества $M \subset \mathbb{C}$ будем полагать

$$
M^{-}=\inf \{\operatorname{Re} z: z \in M\}, \quad M^{+}=\sup \{\operatorname{Re} z: z \in M\} .
$$

УТВЕРЖДЕНИЕ 1. Пусть в условиях леммы 2 функиия $\eta(z)$ голоморфна вне $i$-выпуклого компакта $K \subset U, i$-выпуклый компакт $k \subset D$ связен $u$

$$
k \supset K \cap\left(K-i s_{r}\right) .
$$

Тогда функиия $\gamma(z)$ будет мероморфна с конечным числом полюсов вне компакта $k$.

ДокАЗАТЕЛЬСтво. Установим вначале, что функция $\gamma(z)$ мероморфна в полуплоскостях

$$
\left\{z \in \mathbb{C}: \operatorname{Re} z<k^{-}\right\}, \quad\left\{z \in \mathbb{C}: \operatorname{Re} z>k^{+}\right\} .
$$

Ограничимся случаем правой полуплоскости и обозначим через $\sigma$ точную нижнюю грань чисел $c \geqslant k^{+}$, для которых функция $\gamma(z)$ мероморфна в полуплоскости

$$
\{z \in \mathbb{C}: \operatorname{Re} z>c\} .
$$

Предположим, что $\sigma>k^{+}$.

В таком случае, как несложно показать, прямая

$$
\{z \in \mathbb{C}: \operatorname{Re} z=\sigma\}
$$

будет пересекаться с областью $D$ по непустому интервалу, а пересечение компакта $K$ с этой прямой будет иметь длину, меньшую $s_{r}$, поэтому, очевидно, найдутся числа $a, b, a_{1}, b_{1} \in \mathbb{R}$, для которьй выполнены соотношения

$$
b-a>s_{r}, \quad a<a_{1} \leqslant b_{1}<b-s_{r}, \quad b_{1}-a_{1}<s_{r},
$$

и лучи

$$
\left\{\sigma+i y: y<a_{1}\right\}, \quad\left\{\sigma+i y: y>b_{1}\right\}
$$

не пересекаются с компактом $K$. 
Элементарными рассуждениями можно теперь показать существование чисел $a_{2}, b_{2} \in \mathbb{R}$ таких, что лучи

$$
\left\{\sigma+i y: y<a_{2}\right\}, \quad\left\{\sigma+i y: y>b_{2}\right\}
$$

не пересекаются с компактом $K, \sigma+i a_{2}, \sigma+i\left(b_{2}-s_{r}\right) \in D$, и $b_{2}-a_{2}<s_{r}$, поэтому по лемме 2 функция $\gamma(z)$ будет мероморфно продолжаться из бесконечности по лучам

$$
\left\{\sigma+i y: y<a_{2}\right\}, \quad\left\{\sigma+i y: y>b_{2}-s_{r}\right\} .
$$

Неравенство $\left(b_{2}-a_{2}\right)<s_{r}$ обеспечивает пересечение этих лучей, а мероморфность функции $\gamma(z)$ в полуплоскости

$$
\{z \in \mathbb{C}: \operatorname{Re} z>\sigma\}
$$

гарантирует однозначность функции $\gamma(z)$ на прямой $(3)$.

Но функция $\gamma(z)$ голоморфна вне некоторого компакта, поэтому найдется число $\varepsilon>0$ такое, что эта функция будет мероморфна в полуплоскости

$$
\{z \in \mathbb{C}: \operatorname{Re} z>\sigma-\varepsilon\} .
$$

Противоречие с предположением. Равенство $\sigma=k^{+}$доказано.

Рассмотрим теперь произвольную точку $z_{0} \notin k, z_{0}=x_{0}+i y_{0}, k^{-} \leqslant x_{0} \leqslant k^{+}$. Так как компакт $k$ связен, то его проекция на вещественную ось совпадает с отрезком $\left[k^{-}, k^{+}\right]$, поэтому прямая $\left\{z \in \mathbb{C}: \operatorname{Re} z=x_{0}\right\}$ пересекается с компактом $k$ по некоторому отрезку

$$
\left[x_{0}+i y_{1}, x_{0}+i y_{2}\right],
$$

$y_{1}, y_{2} \in \mathbb{R}, y_{1} \leqslant y_{2}$, а пересечение компакта $K$ с этой прямой будет содержаться в отрезке

$$
\left[x_{0}+i y_{1}, x_{0}+i y_{2}+i s_{r}\right] .
$$

Будем считать, что для точки $z_{0}$ выполнено неравенство $y_{0}<y_{1}$.

По условию функция $\eta(z)$ голоморфна на луче $\left\{x_{0}+i y: y<y_{1}\right\}$ и по лемме 2 функция $\gamma(z)$ будет мероморфно продолжаться из бесконечности по лучу $\left\{x_{0}+i y\right.$ : $\left.y \leqslant y_{0}\right\}$. Отсюда следует существование выпуклой вертикальной полуполосы, уходящей вниз и содержащей точку $z_{0}$, в которой функция $\gamma(z)$ мероморфна.

Аналогично для любой точки, указанной выше, компакта $k$ найдется содержащая ее выпуклая вертикальная полуполоса, уходящая вверх, в которой функция $\gamma(z)$ мероморфна.

Несложно показать, что если две такие полуполосы пересекаются, то они обязательно направлены в одну сторону. Отсюда следует совпадение значений функции $\gamma(z)$ в общей части полуполос, и мероморфность функции $\gamma(z)$ вне компакта $k$ показана.

Допустим теперь, что у функции $\gamma(z)$ бесконечное число полюсов вне компакта $k$. Принимая во внимание лемму 2 , без ограничения общности можно считать, что найдется последовательность

$$
\left\{z_{n} \in D: n \in \mathbb{N}\right\}
$$

ее различных полюсов, для которых функция $\gamma(z)$ голоморфна на лучах

$$
\left\{z \in \mathbb{C}: \operatorname{Re} z=\operatorname{Re} z_{n}, \operatorname{Im} z<\operatorname{Im} z_{n}\right\},
$$

$n \in \mathbb{N}$. Множество (4), очевидно, компактно лежит в области $D$, и по лемме 2 функция $g_{0}(z)$ обращается на нем в нуль. Теорема единственности влечет за собой тождественное равенство этой функции нулю. Противоречие с соотношениями (1).

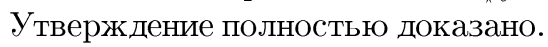


СлеДСТВИЕ. Пусть в условиях утверждения 1 компакт $K$ связен и $k=$ $K \cap\left(K-i s_{r}\right)$. Тогда найдется такой функционал $S_{1} \in H^{*}(D)$ с преобразованием Коши $\gamma_{1}(z)$, что $\mathscr{A}^{*} S_{1}=\mathscr{A}^{*} S$, а функция $\gamma_{1}(z)$ голоморфна вне полосы

$$
\left\{z \in \mathbb{C}: K^{-} \leqslant \operatorname{Re} z \leqslant K^{+}\right\} .
$$

ДокАЗАТЕльСТво. Действительно, так как $k \subset K$, то из утверждения 1 вытекает, что функцию $\gamma(z)$ можно представить в виде

$$
\gamma(z)=\gamma_{1}(z)+\gamma_{2}(z),
$$

где функция $\gamma_{1}(z)$ голоморфна вне полосы $(5)$, а функция $\gamma_{2}(z)$ рациональна и голоморфна на ней, $\gamma_{1}(\infty)=\gamma_{2}(\infty)=0$. Ясно, что особенности этих функций будут лежать во множестве особенностей функции $\gamma(z)$.

Обозначим через $S_{1}$ и $S_{2}$ линейные непрерывные функционалы, преобразование Коши которых совпадает соответственно с функциями $\gamma_{1}(z)$ и $\gamma_{2}(z)$. Понятно, что $S_{1}, S_{2} \in H^{*}(D)$.

Преобразование Коши $\eta_{2}(z)$ функционала $\mathscr{A}^{*} S_{2}$ будет рациональной функцией, голоморфной в полосе (5). С другой стороны, из равенства

$$
\mathscr{A}^{*} S_{2}=\mathscr{A}^{*} S-\mathscr{A}^{*} S_{1}
$$

следует, что все особенности функции $\eta_{2}(z)$ должны лежать в этой полосе. Таким образом, функция $\eta_{2}(z)$ тождественно равна нулю и, следовательно, $\mathscr{A}^{*} S_{2}=0$, поэтому функционал $S_{1}$ искомый.

\section{§ 2. Необходимые условия}

В данном параграфе мы найдем необходимые условия замкнутости образа оператора $\mathscr{A}$.

Лемма 3. Пусть образ оператора $\mathscr{A}$ замкнут, интервал

$$
I=\left\{h_{0}+i y: 0<y<y_{0}\right\}
$$

лежит в области $U, y_{0} \leqslant s_{r}$, и на множестве

$$
\left\{h_{0}+i y: y \in \mathbb{R}\right\} \cap D
$$

функиия $g_{0}(z) g_{r}(z)$ не имеет нулей.

Тогда ядро оператора $\mathscr{A}$ плотно в пространстве $C(I)$.

Здесь через $C(I)$ обозначено пространство непрерывных комплекснозначных функций на интервале $I$ с топологией равномерной сходимости на компактах. 
ДокАЗАТЕЛЬСТво. Так как пространства функций, голоморфных в области, очевидно, являются пространствами $\Phi$ реше, то замкнутость образа оператора $\mathscr{A}$ эквивалентна замкнутости образа сопряженного оператора $\mathscr{A}^{*}$ (см. [4, с. 712]).

Рассмотрим произвольный линейньй непрерывный функционал $T$ на пространстве $C(I)$, аннулируюший ядро оператора $\mathscr{A}$. Ясно, что этот функционал будет непрерывен на пространстве $H(U)$, поэтому из замкнутости образа оператора $\mathscr{A}^{*}$ вытекает сушествование функционала $S \in H^{*}(D)$ со свойством

$$
T=\mathscr{A}^{*} S
$$

(см. $[4$, с. 705$])$.

Обозначим через $\gamma(z)$ и $\eta(z)$ преобразования Коши функционалов $S$ и $T$. Легко видеть, что функция $\eta(z)$ будет голоморфна вне некоторого отрезка $K \subset I$.

Так как $K \cap\left(K-i s_{r}\right)=\varnothing$, то из утверждения 1 вытекает, что функция $\gamma(z)$ рациональна, и по лемме 2 на прямой

$$
\left\{z \in \mathbb{C}: \operatorname{Re} z=\operatorname{Re} h_{0}\right\}
$$

у нее полюсов нет, следовательно, у функции $\eta(z)$ также не будет особенностей на этой прямой.

В таком случае, очевидно, функция $\eta(z)$ голоморфна всюду и, значит, она тождественно равна нулю, следовательно, функционал $T$ нулевой, и теорема Хана-Банаха влечет за собой искомое.

Лемма 4. Пусть образ оператора $\mathscr{A}$ замкнут и для чисел $z_{0} \in \mathbb{C}, m, n \in$ $\mathbb{N}_{0}, \quad 0 \leqslant m \leqslant n \leqslant r, \quad n-m<r$, выполнены соотночения

$$
z_{0}+i s_{m}, z_{0}+i s_{n} \in U \text {. }
$$

Тогда в ядре оператора $\mathscr{A}$ найдутся такие функции $f_{1}, \ldots, f_{n-m+1} \in H(U)$, что определитель

$$
\left|\begin{array}{cccc}
f_{1}\left(z+i s_{m}\right) & f_{1}\left(z+i s_{m+1}\right) & \ldots & f_{1}\left(z+i s_{n}\right) \\
\ldots \ldots \ldots \ldots \ldots \ldots \ldots \ldots \ldots \ldots \ldots \ldots \ldots \ldots \ldots \ldots \ldots \ldots \ldots \ldots \ldots \ldots \ldots \ldots & \ldots \ldots \ldots
\end{array}\right|
$$

отличен от тождественного нуля в окрестности точки $z_{0}$.

ДоКАЗАТЕЛЬСТво. Точка $z_{0}$ принадлежит открытому множеству

$$
\left(U-i s_{m}\right) \cap\left(U-i s_{n}\right) .
$$

В содержащей точку $z_{0}$ связной компоненте этого множества, как легко видеть, найдется точка $h_{0}$, для которой множество (6) не содержит нулей функции $g_{0}(z) g_{r}(z)$.

В таком случае из леммы 3 и теоремы Хана-Банаха вытекает, что в ядре оператора $\mathscr{A}$ сушествует функция $f_{1}$, для которой

$$
f_{1}\left(h_{0}+i s_{m}\right) \neq 0 .
$$


Рассмотрим функционал $T \in C^{*}\left(\left[i s_{m}, i s_{n}\right]\right)$, определенньй по формуле

$$
\langle T, f\rangle=\left|\begin{array}{cc}
f_{1}\left(h_{0}+i s_{m}\right) & f_{1}\left(h_{0}+i s_{m+1}\right) \\
f\left(h_{0}+i s_{m}\right) & f\left(h_{0}+i s_{m+1}\right)
\end{array}\right| .
$$

Это, очевидно, ненулевой функционал, и длина его носителя меньше $s_{r}$.

Из леммы 3 и теоремы Хана-Банаха заключаем, что в ядре оператора $\mathscr{A}$ найдется функция $f_{2}$, для которой

$$
\left|\begin{array}{ll}
f_{1}\left(h_{0}+i s_{m}\right) & f_{1}\left(h_{0}+i s_{m+1}\right) \\
f_{2}\left(h_{0}+i s_{m}\right) & f_{2}\left(h_{0}+i s_{m+1}\right)
\end{array}\right| \neq 0 .
$$

Проведя подобные рассуждения несколько раз, найдем функции $f_{1}, \ldots$ $\ldots, f_{n-m+1} \in \operatorname{Ker} \mathscr{A}$, для которых определитель (7) отличен от нуля в точке $h_{0}$. Ясно, что эти функции искомые.

Приведем теперь основное утверждение данного параграфа.

Tеорема 1. Для замкнутости образа оператора $\mathscr{A}$ необходимы следующие условия:

а) любая точка мнозсеств

$$
\partial D \cap\left(U-i s_{1}\right) \cap\left(U-i s_{r}\right), \quad \partial D \cap U \cap\left(U-i s_{r-1}\right)
$$

обладает окрестностью с конечным числом нулей соответственно функций $g_{0}(z)$ u $g_{r}(z)$

б) любая точка множества

$$
\partial D \cap\left(U-i s_{1}\right) \cap\left(U-i s_{r-1}\right)
$$

обладает окрестностью с конечным числом общих нулей функиий $g_{0}(z)$ $u g_{r}(z)$.

ДокАЗАТЕЛЬСтво. Предположим, что утверждение не верно, и найдется, например, последовательность точек $\left\{z_{n} \in D: n \in \mathbb{N}\right\}$, сходящаяся к точке $z_{0}$, причем

$$
z_{0} \in\left(U-i s_{1}\right) \cap\left(U-i s_{r}\right), \quad g_{0}\left(z_{n}\right)=0, \quad n \in \mathbb{N} .
$$

В таком случае для любых функций $f_{1}, \ldots, f_{r} \in \operatorname{Ker} \mathscr{A}$ имеем

$$
\sum_{j=1}^{r} g_{j}\left(z_{n}\right) f_{k}\left(z_{n}+i s_{j}\right)=0, \quad k=1, \ldots, r, \quad n \in \mathbb{N} .
$$

Числа $g_{1}\left(z_{n}\right), \ldots, g_{r}\left(z_{n}\right)$ одновременно в нуль не обрашаются, поэтому из последних равенств заключаем

$$
\left|\begin{array}{ccc}
f_{1}\left(z_{n}+i s_{1}\right) & \ldots & f_{1}\left(z_{n}+i s_{r}\right) \\
\ldots \ldots \ldots \ldots \ldots \ldots \ldots \ldots \ldots \\
f_{r}\left(z_{n}+i s_{1}\right) & \ldots & f_{r}\left(z_{n}+i s_{r}\right)
\end{array}\right|=0, \quad n \in \mathbb{N} .
$$

Но функция

$$
\left|\begin{array}{ccc}
f_{1}\left(z_{n}+i s_{1}\right) & \ldots & f_{1}\left(z_{n}+i s_{r}\right) \\
\ldots \ldots \ldots \ldots \ldots \ldots \ldots \ldots \ldots \\
f_{r}\left(z_{n}+i s_{1}\right) & \ldots & f_{r}\left(z_{n}+i s_{r}\right)
\end{array}\right|
$$

голоморфна в точке $z_{0}$ и, следовательно, будет тождественно равна нулю в некоторой окрестности этой точки, что противоречит лемме 4.

Аналогичные рассуждения можно провести и для оставшихся двух множеств. Теорема доказана. 


\section{§ 3. Достаточные условия}

В данном параграфе мы найдем условия, при которых образ оператора $\mathscr{A}$ будет замкнут.

ЛЕмма 5. Пусть функиии $g_{0}(z)$ и $g_{r}(z)$ имеют конечное число нулей $в$ области $D$, а множество

$$
\bigcup_{j=1}^{r-1}\left(D+i s_{j}\right)
$$

компактно лежит в области $U$.

Тогда образ оператора $\mathscr{A}$ замкнут и имеет конечную коразмерность $в$ пространстве $H(D)$.

ДокаЗАТЕЛЬСтво. Разберем вначале несколько частных случаев.

Допустим, что оператор $\mathscr{A}_{1}$ определен по формуле

$$
\left(\mathscr{A}_{1} f\right)(z)=f\left(z+i s_{r}\right)-f(z), \quad f \in H(U),
$$

и установим эпиморфность этого оператора.

Как хорошо известно в теории операторов свертки, оператор $\mathscr{A}_{1}$ будет эпиморфен, если имеет место импликация

$$
\left(S \in H^{*}(\mathbb{C}), \mathscr{A}_{1}^{*} S \in H^{*}(U)\right) \Rightarrow S \in H^{*}(D) .
$$

Пусть компакт $K \subset U$ содержит все особенности преобразования Коши $\eta(z)$ функционала $\mathscr{A}_{1}^{*} S$. Воспользовавшись леммой 2 статьи автора [3], найдем такие гладкие вешественные функции $\alpha(x)$ и $\beta(x)$ на некотором отрезке $\left[a_{0}, b_{0}\right]$, что $i$-выпуклый компакт

$$
K_{1}=\left\{x+i y: \alpha(x) \leqslant y \leqslant \beta(x), x \in\left[a_{0}, b_{0}\right]\right\}
$$

удовлетворяет соотношениям

$$
K \subset K_{1} \subset U
$$

а компакт

$$
k=K_{1} \cap\left(K_{1}-i s_{r}\right)
$$

связен и, очевидно, лежит в области $D$.

В таком случае утверждение 1 и лемма 2 гарантируют голоморфность преобразования Коши функционала $S$ вне компакта $k$. Но это и означает, что $S \in H^{*}(D)$, и эпиморфность оператора $\mathscr{A}_{1}$ показана.

Рассмотри теперь оператор $\mathscr{A}_{2}$, определенный по формуле

$$
\left(\mathscr{A}_{2} f\right)(z)=g_{0}(z) f(z)+g_{r}(z) f\left(z+i s_{r}\right), \quad f \in H(U),
$$

и докажем существование в ядре этого оператора функции $F \in H(U)$ с конечным числом нулей в области $D$.

Если функции $f_{1}, f_{2} \in H(U)$ удовлетворяют уравнениям

$$
p_{l}(z) f_{l}(z)+q_{l}(z) f_{l}\left(z+i s_{r}\right)=0, \quad l=1,2,
$$


для некоторых функций $p_{l}, q_{l} \in H(D), l=1,2$, то, как легко видеть, функция

$$
f(z)=e^{\pi s_{r}^{-1} z} f_{1}(z) f_{2}(z)
$$

будет решением уравнения

$$
p_{1}(z) p_{2}(z) f(z)+q_{1}(z) q_{2}(z) f\left(z+i s_{r}\right)=0 .
$$

Эти соображения вместе с условием конечности числа нулей функций $g_{0}(z)$ и $g_{r}(z)$ показывают, что для построения функции $F(z)$ достаточно найти голоморфные в области $U$ с конечным числом корней в области $D$ решения уравнений

$$
\begin{aligned}
\left(z+z_{1}\right) f(z)+f\left(z+i s_{r}\right) & =0 \\
f(z)+\left(z+z_{2}\right) f\left(z+i s_{r}\right) & =0 \\
e^{\varphi(z)} f(z)+f\left(z+i s_{r}\right) & =0
\end{aligned}
$$

где $z_{1}, z_{2} \in \mathbb{C}, \varphi \in H(D)$.

Для первых двух уравнений несложно подобрать целье решения вида

$$
\frac{e^{c z}}{\Gamma(a z+b)}
$$

$a, b, c \in \mathbb{C}$. Так как множество (8) по условию ограничено, эти функции действительно будут иметь конечное число нулей в области $D$.

Займемся теперь уравнением (9). Из эпиморфности оператора $\mathscr{A}_{1}$ вытекает, что найдется функция $C(z) \in H(U)$, удовлетворяющая уравнению

$$
C\left(z+i s_{r}\right)-C(z)=\pi i+\varphi(z) .
$$

В таком случае, очевидно, функция $\exp C(z)$ будет решением уравнения (9), не имеющим нулей, поэтому существование искомой функции $F(z)$ установлено.

Для произвольной функции $f \in H(U)$ имеем

$$
g_{0}(z) f(z) F(z)+g_{r}(z) f\left(z+i s_{r}\right) F\left(z+i s_{r}\right)=g_{0}(z) F(z)\left[f(z)-f\left(z+i s_{r}\right)\right] .
$$

Это соотношение вместе с эпиморфностью оператора $\mathscr{A}_{1}$ означает, что образ оператора $\mathscr{A}_{2}$ содержит пространство голоморфных функций в области $D$, которые делятся на функцию $g_{0}(z) F(z)$. Но эта функция имеет конечное число нулей в области $D$, поэтому указанное пространство является замкнутым подпространством конечной коразмерности в $H(D)$.

В таком случае, как легко видеть, образ оператора $\mathscr{A}_{2}$ будет равен сумме этого замкнутого подпространства конечной коразмерности и некоторого конечномерного подпространства $H(D)$, поэтому образ замкнут (см. [5, c. 41]) и, очевидно, конечной коразмерности в пространстве $H(D)$.

Рассмотрим теперь оператор

$$
\mathscr{B}: H(U) \rightarrow H(D),
$$


определенный по формуле

$$
(\mathscr{B} f)(z)=\sum_{j=1}^{r-1} g_{j}(z) f\left(z+i s_{j}\right), \quad f \in H(U)
$$

и докажем его компактность.

Действительно, совокупность всех функций пространства $H(U)$, модуль которых не превосходит единицы на множестве (8), будет окрестностью нуля, а образ этой совокупности при отображении $\mathscr{B}$, очевидно, будет предкомпактом в пространстве $H(D)$.

В таком случае из леммы 1 статьи автора [6] вытекает, что образ оператора $\left(\mathscr{A}_{2}+\mathscr{B}\right)$ будет замкнут и конечной коразмерности в пространстве $H(D)$. Лемма полностью доказана.

ЛЕмма 6. Пусть для любого связного компакта $K \subset U$ и последовательности функиионалов $\left\{R_{n} \in H^{*}(D): n \in \mathbb{N}\right\}$ таких, что компакт $K$ является определяющим множсеством функционала $\mathscr{A}^{*} R_{n}$, а преобразование Коши функиионала $R_{n}$ голоморфно вне полосы $(5), n \in \mathbb{N}$, найдется компакт $k \subset D$, являющийся определяющим множеством функиионалов $R_{n}, \quad n \in \mathbb{N}$.

Тогда образ оператора $\mathscr{A}$ замкнут в пространстве $H(D)$.

ДокАЗАТЕльство. Как уже отмечалось, замкнутость образа оператора $\mathscr{A}$ эквивалентна замкнутости образа сопряженного оператора $\mathscr{A}^{*}$. Пространство $H^{*}(U)$ есть $L N^{*}$-пространство, поэтому замкнутость его подпространств эквивалентна их секвенциальной замкнутости (см. [7]).

В таком случае рассмотрим последовательность $\left\{R_{n}: n \in \mathbb{N}\right\}$ функционалов пространства $H^{*}(D)$ такую, что для некоторого функционала $T \in H^{*}(U)$

$$
\mathscr{A}^{*} R_{n} \rightarrow T
$$

в топологии пространства $H^{*}(U)$. Как несложно показать, найдутся связньй компакт $K \subset U$ и число $c>0$, обеспечивающие неравенства

$$
\left|\left\langle\mathscr{A}^{*} R_{n}, f\right\rangle\right| \leqslant c \max _{t \in K}|f(t)|, \quad f \in H(U), \quad n \in \mathbb{N} .
$$

Из этих неравенств следует, что компакт $K$ является определяюшим множеством функционалов $\mathscr{A}^{*} R_{n}$ для всех $n \in \mathbb{N}$.

Принимая во внимание следствие утверждения 1 , преобразование Коши функционала $R_{n}$ можно считать голоморфным вне полосы (5), $n \in \mathbb{N}$.

По условию существует компакт $k \subset D$, являющийся определяющим множеством функционалов $R_{n}, n \in \mathbb{N}$. Положим

$$
K_{1}=K \cup\left(k+\left[0, i s_{r}\right]\right)
$$

Как несложно убедиться, компакт $K_{1}$ лежит в области $U$ и удовлетворяет соотношению

$$
K_{1} \cap\left(K_{1}-i s_{r}\right) \supset k .
$$


Из упомянутой вьше леммы 2 статьи [3] вытекает сушествование гладких функций $\alpha(x)$ и $\beta(x)$ на некотором отрезке $\left[a_{0}, b_{0}\right]$ со свойствами:

$$
\begin{gathered}
U \supset\left\{x+i y: \alpha(x) \leqslant y \leqslant \beta(x), \quad x \in\left(a_{0}, b_{0}\right)\right\}, \\
K_{1} \subset\left\{x+i y: \alpha(x)<y<\beta(x), \quad x \in\left[a_{0}, b_{0}\right]\right\}, \\
\left\{x \in\left[a_{0}, b_{0}\right]: \alpha(x)+j s_{r}<\beta(x)\right\}=\left(a_{j}, b_{j}\right), \quad j=0,1,
\end{gathered}
$$

где $a_{0}<a_{1}<b_{1}<b_{0}$.

Из этих соотношений заключаем, что множество

$$
U_{1}=\left\{x+i y: \alpha(x)<y<\beta(x), \quad x \in\left(a_{0}, b_{0}\right)\right\}
$$

является областью, компактно лежащей в области $U, K_{1} \subset U_{1}$, множество

$$
D_{1}=U_{1} \cap\left(U_{1}-i s_{r}\right)
$$

связно и компактно лежит в области $D$. Область $U_{1}$, очевидно, $i$-выпукла.

Как несложно показать, для области $D_{1}$ имеет место представление

$$
D_{1}=\left\{x+i y: \alpha(x)<y<\beta(x)-s_{r}, \quad x \in\left(a_{1}, b_{1}\right)\right\},
$$

поэтому замыкание области $D_{1}+i s_{j}$ совпадает с множеством

$$
\left\{x+i y: \alpha(x)+s_{j} \leqslant y \leqslant \beta(x)-s_{r}+s_{j}, \quad x \in\left[a_{1}, b_{1}\right]\right\},
$$

$j=1, \ldots, r-1$.

Эти представления показывают, что множество

$$
\bigcup_{j=1}^{r-1}\left(D_{1}+i s_{j}\right)
$$

компактно лежит в области $U_{1}$.

Оператор $\mathscr{A}$, очевидно, продолжается по непрерывности до оператора

$$
\mathscr{A}_{1}: H\left(U_{1}\right) \rightarrow H\left(D_{1}\right)
$$

определенного той же формулой, а функции $g_{0}(z)$ и $g_{r}(z)$ имеют в области $D_{1}$ конечное число нулей. Из леммы 5 следует, что у оператора $\mathscr{A}_{1}$ и, значит, у $\mathscr{A}_{1}^{*}$ образ замкнут.

Как легко убедиться, функционалы $R_{n}$ будут принадлежать пространству $H^{*}\left(D_{1}\right), n \in \mathbb{N}$, и $\mathscr{A}_{1}^{*} R_{n} \rightarrow T$ в топологии пространства $H^{*}\left(U_{1}\right)$. Из вьшесказанного заключаем, что найдется функционал $R \in H^{*}\left(D_{1}\right)$, для которого

$$
\mathscr{A}_{1}^{*} R=T \text {. }
$$

Но, очевидно, $\mathscr{A}_{1}^{*} R=\mathscr{A}^{*} R$, что и доказывает искомое. 
УТВЕРЖДЕНИЕ 2. Пусть для любого компакта $K \subset U$ и последовательности функционалов $S_{n} \in H^{*}(D)$ с преобразованиями Коши $\gamma_{n}(z), n \in \mathbb{N}$, таких, что компакты $K u k=K \cap\left(K-i s_{r}\right) \quad i$-выпукльи и связны, компакт $K$ является определяющим множеством всех функционалов $\mathscr{A}^{*} S_{n}$, а функции $\gamma_{n}(z)$ рачиональны с полюсами на одной прямой

$$
\left\{z \in \mathbb{C}: \operatorname{Re} z=x_{n}\right\}
$$

$n \in \mathbb{N}$, причем эти прямые лежат в полосе (5), найдется компакт области $D$, содержащий полюса всех функиий $\gamma_{n}(z)$.

Тогда образ оператора $\mathscr{A}$ замкнут.

ДокаЗАТЕЛЬСтво. Рассмотрим компакт $K \subset U$ и последовательность функционалов $R_{n} \in H^{*}(D)$ с преобразованиями Коши $\chi_{n}(z)$, для которых выполнены условия леммы $6, n \in \mathbb{N}$. Можно считать, что компакты $K$ и $k=K \cap\left(K-i s_{r}\right)$ $i$-выпуклы и связны.

Используя утверждение 1 , несложно представить функцию $\chi_{n}(z)$ в виде

$$
\chi_{n}(z)=\chi_{n}^{1}(z)+\chi_{n}^{2}(z)
$$

где функция $\chi_{n}^{1}$ голоморфна вне компакта $k$, а функция $\chi_{n}^{2}$ рациональна с полюсами в полосе $(5), \chi_{n}^{1}(\infty)=\chi_{n}^{2}(\infty)=0, n \in \mathbb{N}$.

Обозначим через $R_{n}^{1}$ и $R_{n}^{2}$ линейные непрерывные функционалы, преобразования Коши которых совпадают соответственно с функциями $\chi_{n}^{1}(z)$ и $\chi_{n}^{2}(z), n \in \mathbb{N}$. Ясно, что $R_{n}^{1}, R_{n}^{2} \in H^{*}(D)$, поэтому определены функционалы $\mathscr{A}^{*} R_{n}^{1}$ и $\mathscr{A}^{*} R_{n}^{2}$, чьи преобразования Коши обозначим через $\mu_{n}^{1}(z)$ и $\mu_{n}^{2}(z), n \in \mathbb{N}$.

Как несложно убедиться, имеют место включения

$$
k+i s_{j} \subset K, \quad j=0, \ldots, r,
$$

и, следовательно, функция $\mu_{n}^{1}(z)$ будет голоморфна вне компакта $K$, что влечет за собой и голоморфность функции $\mu_{n}^{2}(z)$ вне этого компакта, $n \in \mathbb{N}$.

Пусть $\chi(z)$ - рациональная функция, построенная по всем полюсам некоторой функции $\chi_{n}^{2}(z)$, лежашим на одной вертикальной прямой, $\chi(\infty)=0$, a $R \in H^{*}(D)$ функционал с преобразованием Коши $\chi(z)$. Нетрудно убедиться, что преобразование Коши функционала $\mathscr{A}^{*} R$ будет голоморфно вне компакта $K$, а всего таких функционалов $R$ не более счетного множества. В таком случае по условию найдется компакт $k_{1} \subset D$, содержащий особенности всех функций $\chi_{n}^{2}$.

Суммируя вышесказанное, получим, что компакт $\left(k \cup k_{1}\right)$ является определяющим множеством функционала $R_{n}, n \in \mathbb{N}$, и искомое следует из леммы 6 .

Сформулируем теперь основной результат данного параграфа.

ТЕОрема 2. Пусть для любого числа $c \in\left(U^{-}, U^{+}\right) \cap\left\{D^{-}, D^{+}\right\}$найдутся числа $y_{0} \in \mathbb{R} u \varepsilon>0$ со свойствами:

а) лучи $\left\{c+i y: y \leqslant y_{0}\right\},\left\{c+i y+i s_{r}: y \geqslant y_{0}\right\}$ не пересекаются с областью $U$;

б) множества

$$
\begin{aligned}
& \left\{x+i y:|x-c|<\varepsilon, \quad y \leqslant y_{0}\right\} \cap D \\
& \left\{x+i y:|x-c|<\varepsilon, \quad y \geqslant y_{0}\right\} \cap D
\end{aligned}
$$


не содержат нулей соответственно функиий $g_{0}(z)$ u $g_{r}(z)$.

Пусть, далее, для любого компакта $k \subset D$ множества

$$
D \cap \bigcup_{y \leqslant 0}(k+i y), \quad D \cap \bigcup_{y \geqslant 0}(k+i y)
$$

содержат конечное число нулей соответственно функций $g_{0}(z)$ u $g_{r}(z)$.

Тогда образ оператора $\mathscr{A}$ замкнут.

ДокАЗАТЕЛЬСТво. Рассмотрим компакт $K \subset U$ и последовательность функционалов $\left\{S_{n} \in H^{*}(D): n \in \mathbb{N}\right\}$, удовлетворяющие условиям утверждения 2 , и установим существование чисел $a, b \in \mathbb{R}, D^{-}<a<b<D^{+}$, таких, что $a \leqslant x_{n} \leqslant b$, $n \in \mathbb{N}$.

Действительно, в противном случае без ограничения общности можно считать выполненными соотношения $x_{n}>k^{+}, n \in \mathbb{N}$, и

$$
\lim _{n \rightarrow \infty} x_{n}=D^{+} \text {. }
$$

Как несложно видеть, это равенство обеспечивает неравенство $D^{+} \leqslant K^{+}$и, следовательно,

$$
D^{+} \in\left(U^{-}, U^{+}\right) .
$$

Обозначим через $z_{n}=x_{n}+i y_{n}$ самьй нижний полюс функции $\gamma_{n}(z)$ на прямой $(10)$, а через $\left(z_{n}+i t_{n}\right)$ - самьй верхний, $n \in \mathbb{N}$. В силу неравенства $x_{n}>k^{+}$ пересечение компакта $K$ с указанной прямой имеет длину, меньшую $s_{r}$, поэтому один из лучей

$$
\left\{z_{n}+i y: y \leqslant 0\right\}, \quad\left\{z_{n}+i y+i s_{r}: y \geqslant 0\right\}
$$

не пересекается с этим компактом, $n \in \mathbb{N}$. Для определенности будем считать, что для всех $n \in \mathbb{N}$ этим свойством обладает первый луч, и по лемме 2

$$
g_{0}\left(z_{n}\right)=0, \quad n \in \mathbb{N} .
$$

По условию для числа $c=D^{+}$найдутся числа $y_{0} \in \mathbb{R}$ и $\varepsilon>0$ со свойствами а) и б). Мы можем считать, что $\left|x_{n}-c\right|<\varepsilon, n \in \mathbb{N}$. В таком случае из свойства б) вытекает неравенство $y_{n}>y_{0}, n \in \mathbb{N}$, поэтому, используя свойство а), несложно показать, что второй из вышеприведенных лучей не будет пересекаться с компактом $K$ для достаточно больших $n$.

Следовательно, по лемме 2

$$
g_{r}\left(z_{n}+i t_{n}\right)=0, \quad n \geqslant n_{0} .
$$

Противоречие со свойством а) и неравенством $y_{n}>y_{0}, n \in \mathbb{N}$. Наличие искомых чисел $a$ и $b$ установлено.

Очевидно, в области $D$ найдется связный компакт $k_{1} \supset k$, проекция которого на вешественную ось совпадает с отрезком $[a, b]$. Так как у функций $g_{0}(z)$ и $g_{r}(z)$ конечное число нулей соответственно ниже и вьше компакта $k_{1}$, то, соединив эти нули с компактом $k_{1}$ вертикальными отрезками, получим компакт $k_{2} \subset D$.

Из леммы 2 следует, что полюса всех функций $\gamma_{n}(z)$ будут лежать в компакте $k_{2}$, и искомое вытекает из утверждения 2. 
СлЕДСТвиЕ. Пусть

$$
U=D+\left[0, i s_{r}\right]
$$

а множества (11) содержат конечное число нулей соответственно функиий $g_{0}(z) u g_{r}(z)$.

Тогда образ оператора $\mathscr{A}$ замкнут.

Действительно, в данном случае, очевидно, $D^{ \pm}=U^{ \pm}$, поэтому множество

$$
\left(U^{-}, U^{+}\right) \cap\left\{D^{-}, D^{+}\right\}
$$

пустое, и искомое вытекает из теоремы 2.

\section{§ 4. Необходимые и достаточные условия}

В данном параграфе мы опишем класс областей комплексной плоскости, для которых имеется критерий замкнутости образа оператора $\mathscr{A}$ в терминах нулей его крайних коэффициентов.

Для множества $M \subset \mathbb{C}$ введем следующие функции:

$$
\begin{aligned}
& \alpha(x, M):=\inf \{y \in \mathbb{R}: x+i y \in M\}, \\
& \beta(x, M):=\sup \{y \in \mathbb{R}: x+i y \in M\} .
\end{aligned}
$$

Если $M \subset \mathbb{C}$ - область, то легко показать, что функции $\alpha(x, M)$ и $\beta(x, M)$ будут полунепрерывны соответственно сверху и снизу в каждой точке интервала $\left(M^{-}, M^{+}\right)$.

$i$-вьпуклая область $U \subset \mathbb{C}$, очевидно, представляется в виде

$$
U=\left\{x+i y: \alpha(x, U)<y<\beta(x, U), \quad x \in\left(U^{-}, U^{+}\right)\right\},
$$

а область $D$ запишется в виде

$$
D=\left\{x+i y: \alpha(x, U)<y<\beta(x, U)-s_{r}, \quad x \in\left(D^{-}, D^{+}\right)\right\} .
$$

Сформулируем основной результат данного параграфа.

ТЕорема 3. Пусть для любой точки $x_{0} \in\left[D^{-}, D^{+}\right] \cap\left(U^{-}, U^{+}\right)$имеют место соотношения

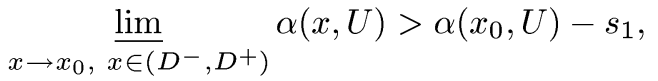

$$
\begin{aligned}
& \varlimsup_{x \rightarrow x_{0},} \varlimsup_{x \in\left(D^{-}, D^{+}\right)} \beta(x, U)<\beta\left(x_{0}, U\right)+s_{r}-s_{r-1} .
\end{aligned}
$$

Тогда замкнутость образа оператора $\mathscr{A}$ әквивалентна выполнению условий а) и б) теоремы 1. 
ДоКАЗАТЕЛЬСТво. Принимая во внимание теорему 1 , нам надо показать, что из условий а) и б) этой теоремы вытекает замкнутость образа оператора $\mathscr{A}$.

Начало наших рассуждений будет таким же, как при доказательстве теоремы 2 до равенства (12). Далее продолжаем с этого момента.

Точки $z_{n}$ и $z_{n}+i t_{n}$ лежат в области $D$, поэтому

$$
\alpha\left(x_{n}, U\right)<y_{n}<\beta\left(x_{n}, U\right)-s_{r}, \quad \alpha\left(x_{n}, U\right)<y_{n}+t_{n}<\beta\left(x_{n}, U\right)-s_{r},
$$

и из условий на функции $\alpha(x, U)$ и $\beta(x, U)$ заключаем, что

$$
\begin{gathered}
\alpha\left(D^{+}, U\right)-s_{1}<\underline{\lim _{n \rightarrow \infty}} y_{n}, \\
\varlimsup_{n \rightarrow \infty}\left(y_{n}+t_{n}\right)<\beta\left(D^{+}, U\right)-s_{r-1} .
\end{gathered}
$$

Так как $t_{n} \geqslant 0, n \in \mathbb{N}$, то из последних неравенств вытекает ограниченность последовательностей $\left\{y_{n}: n \in \mathbb{N}\right\}$ и $\left\{t_{n}: n \in \mathbb{N}\right\}$. Можно считать их сходяшимися:

$$
y_{n} \rightarrow y_{0}, \quad t_{n} \rightarrow t_{0},
$$

и, следовательно,

$$
\begin{gathered}
\alpha\left(D^{+}, U\right)-s_{1}<y_{0}, \\
y_{0}+t_{0}<\beta\left(D^{+}, U\right)-s_{r-1} .
\end{gathered}
$$

Точка $z_{0}=D^{+}+i y_{0}$ является предельной для нулей функции $g_{0}(z)$, поэтому из условия а) получаем

$$
z_{0} \notin\left(U-i s_{1}\right) \cap\left(U-i s_{r}\right) .
$$

Как легко показать, последнее множество запишется в виде

$$
\left\{x+i y: \alpha(x, U)-s_{1}<y<\beta(x, U)-s_{r}\right\},
$$

и из соотношений (13) и (15) вытекает неравенство

$$
y_{0}+s_{r} \geqslant \beta\left(D^{+}, U\right) .
$$

Используя представление из леммы 1

$$
\eta_{n}(z)=\sum_{j=0}^{r} \frac{1}{2 \pi i} \int_{C} \frac{\gamma_{n}(t) g_{j}(t)}{z-t-i s_{j}} d t
$$

несложно показать, что на прямой (10) последнее слагаемое правой части голоморфно вне отрезка

$$
\left[z_{n}+i s_{r}, z_{n}+i t_{n}+i s_{r}\right]
$$

а остальные слагаемые голоморфны вне отрезка

$$
\left[z_{n}, z_{n}+i t_{n}+i s_{r-1}\right],
$$

$n \in \mathbb{N}$. 
Из неравенства (16) следует, что точка $\left(z_{0}+i s_{r}\right)$ лежит вьше области $U$, поэтому, начиная с некоторого номера $n \in \mathbb{N}$, точки $\left(z_{n}+i s_{r}\right)$ будут лежать вьше компакта $K$, и, значит, отрезок (17) не пересекается с компактом $K$ для таких $n$, и, следовательно, функция $\eta_{n}(z)$ будет голоморфной на этом отрезке.

Из неравенств (14) и (16) заключаем, что для достаточно больших $n$ отрезки (17) и (18) не пересекаются, поэтому последнее слагаемое в правой части представления функции $\eta_{n}(z)$ будет голоморфно на отрезке (17) для таких $n$.

Воспользовавшись формулой Сохоцкого-Племеля, несложно показать, что функция $\gamma_{n}(z) g_{r}(z)$ будет голоморфна на отрезке $\left[z_{n}, z_{n}+i t_{n}\right]$, и, значит, $g_{r}\left(z_{n}\right)=$ 0 , начиная с некоторого момента.

Таким образом, точка $z_{0}$ является предельной для общих нулей функций $g_{0}(z)$ и $g_{r}(z)$. Но неравенства (13) и (14) обеспечивают включение

$$
z_{0} \in\left(U-i s_{1}\right) \cap\left(U-i s_{r-1}\right),
$$

что противоречит условию б), и существование чисел $a, b \in \mathbb{R}$ с нужными свойствами установлено.

Как несложно убедиться, найдется связный $i$-выпуклый компакт $k_{1} \subset D, k \subset k_{1}$, проекция которого на вешественную ось совпадает с отрезком $[a, b]$. Докажем, что множества

$$
D \cap \bigcup_{t \leqslant 0}\left(k_{1}+i t\right), \quad D \bigcap_{t \geqslant 0}\left(k_{1}+i t\right)
$$

содержат конечное число нулей соответственно функций $g_{0}(z)$ и $g_{r}(z)$.

Действительно, допустим, что существует последовательность

$$
w_{n} \in D \cap \bigcup_{t \leqslant 0}\left(k_{1}+i t\right)
$$

различных нулей функции $g_{0}(z), w_{n}=\zeta_{n}+i \tau_{n}$, где $\zeta_{n} \in k_{1}, \tau_{n} \leqslant 0, n \in \mathbb{N}$. Последовательность $\left\{\zeta_{n}: n \in \mathbb{N}\right\}$ можно считать сходящейся:

$$
\zeta_{n} \rightarrow \zeta_{0}=x_{0}+i y_{0}, \quad x_{0}, y_{0} \in \mathbb{R} .
$$

Ясно, что $\zeta_{0} \in D$.

Как и вьше, из условия на функцию $\alpha(x, U)$ найдем

$$
\alpha\left(x_{0}, U\right)-s_{1}<y_{0}+\underset{n \rightarrow \infty}{\lim } \tau_{n}
$$

и, значит, последовательность $\left\{\tau_{n}\right\}$ ограничена, и ее можно считать сходящейся:

$$
\tau_{n} \rightarrow \tau_{0},
$$

так что

$$
\alpha\left(x_{0}, U\right)-s_{1}<y_{0}+\tau_{0} .
$$

Из включения $\zeta_{0} \in D$ вытекает неравенство

$$
y_{0}<\beta\left(x_{0}, U\right)-s_{r},
$$


поэтому и

$$
y_{0}+\tau_{0}<\beta\left(x_{0}, U\right)-s_{r}
$$

Суммируя вышесказанное, получим, что точка $\zeta_{0}+i \tau_{0}$ является предельной для нулей функции $g_{0}(z)$ и

$$
\zeta_{0}+i \tau_{0} \in\left(U-i s_{1}\right) \cap\left(U-i s_{r}\right)
$$

Противоречие с условием а). Конечность числа интересующих нас нулей установлена.

Окончание доказательства будет таким же, как и в теореме 2.

Докажем теперь, что условия теоремы 3 нельзя ослабить.

УТВЕРЖДЕНИЕ 3. Зафиксируем числа $a, b, c \in \mathbb{R}, a, b>0, a+b<c, u$ предположим, что для любого числа $r \in \mathbb{N}, r \geqslant 3$, произвольный разностный оператор $\mathscr{A}$, удовлетворяющий условиям а) и б) теоремь 1 и соотношениям

$$
s_{1} \geqslant a, \quad s_{r}-s_{r-1} \geqslant b, \quad s_{r}=c
$$

имеет замкнутый образ в пространстве $H(D)$.

Тогда для области $U$ будут выполнены неравенства

$$
\begin{aligned}
\varliminf_{x \rightarrow x_{0}}, & \varlimsup_{x \in\left(D^{-}, D^{+}\right)} \alpha(x, U)>\alpha\left(x_{0}, U\right)-a, \\
\varlimsup_{x \rightarrow x_{0},} & \left.\beta(x, U)<\beta\left(x_{0}, U\right)+b . D^{+}\right)
\end{aligned}
$$

ДоказАТЕльство. Допустим, что это не верно, и в некоторой точке $x_{0} \in$ $\left(U^{-}, U^{+}\right) \cap\left[D^{-}, D^{+}\right]$имеет место, скажем, соотношение

$$
\varliminf_{x \rightarrow x_{0},} \lim _{x \in\left(D^{-}, D^{+}\right)} \alpha(x, U) \leqslant \alpha\left(x_{0}, U\right)-a
$$

Разберем вначале случай

$$
\alpha\left(x_{0}, U\right)=-\infty
$$

В этой ситуации, очевидно, существует точка $z_{0}=x_{0}+i y_{0}$ такая, что луч $\left\{z_{0}+i t\right.$ : $t \leqslant 0\}$ целиком лежит в области $D$. Как несложно убедиться, найдутся числа $k, m, n \in \mathbb{N}$, для которых выполнены неравенства

$$
\frac{k c}{n}>a, \quad c-\frac{m c}{n}>b, \quad k<m
$$

Положим

$$
\begin{gathered}
r=3, \quad s_{1}=\frac{k c}{n}, \quad s_{2}=\frac{m c}{n}, \quad s_{3}=c \\
g_{0}(z)=g_{1}(z)=g_{2}(z)=\sin \left(\frac{\pi i n\left(z-z_{0}\right)}{c}\right), \quad g_{3}(z)=1 .
\end{gathered}
$$


Ясно, что для оператора

$$
(\mathscr{A} f)(z)=\sum_{j=0}^{3} g_{j}(z) f\left(z+i s_{j}\right), \quad f \in H(U),
$$

будут выполнены все необходимые условия. Докажем, что образ этого оператора не замкнут.

Действительно, если $f \in \operatorname{Ker} \mathscr{A}$, то, очевидно,

$$
f\left(z_{0}+\frac{i l c}{n}\right)=0, \quad l \in \mathbb{Z}, \quad l \leqslant n .
$$

Так как $k, m<n$, то, дифференцируя тождество $(\mathscr{A} f)(z)=0$ и подставляя значения $z=z_{0}+i l c / n, l \in \mathbb{Z}, l \leqslant 0$, найдем

$$
f^{\prime}\left(z_{0}+\frac{i l c}{n}\right)=0, \quad l \in \mathbb{Z}, \quad l \leqslant n .
$$

Рассуждая подобным образом дальше, получим тождественное равенство нулю функции $f$. В таком случае из леммы 4 вытекает, что образ оператора $\mathscr{A}$ не замкнут.

Пусть теперь

$$
\alpha\left(x_{0}, U\right)>-\infty .
$$

Как несложно показать, в этой ситуации найдется последовательность точек $z_{n}=$ $x_{n}+i y_{n} \in D$, для которой

$$
x_{n} \rightarrow x_{0}, \quad y_{n} \rightarrow y_{0}=\alpha\left(x_{0}, U\right)-a .
$$

Ясно, что точки $z_{0}=x_{0}+i y_{0}$ и $z_{0}+i a$ лежат на гранище областей $U$ и $D$.

Из определения числа $\alpha\left(x_{0}, U\right)$ следует существование числа $\varepsilon, 0<\varepsilon<c-a-b$, для которого имеет место включение

$$
x_{0}+i \alpha\left(x_{0}, U\right)+i \varepsilon \in U .
$$

Пусть $r=4, s_{1}=a, s_{2}=a+\varepsilon, s_{3}=c-b, s_{4}=c$, функции $g_{0}, g_{1}, g_{3}, g_{4} \in H(D)$ обрашаются в нуль только в точках $z_{n}, n \in \mathbb{N}$, а функция $g_{2}$ в них отлична от нуля.

Точка $z_{0}$ не принадлежит ни одному из множеств условий а) и б) теоремы 1 , поэтому для оператора

$$
(\mathscr{A} f)(z)=\sum_{j=0}^{4} g_{j}(z) f\left(z+i s_{j}\right), \quad f \in H(D),
$$

будут выполнены все необходимые требования.

Для функции $f \in \operatorname{Ker} \mathscr{A}$ имеем

$$
f\left(z_{n}+i s_{2}\right)=0, \quad n \in \mathbb{N} .
$$

Но точка $z_{0}+i s_{2}$ лежит в области $U$, поэтому $f \equiv 0$, и образ оператора $\mathscr{A}$ не замкнут. Утверждение полностью доказано. 
СлЕДСТВИЕ. Пусть число с $>0$ и область $U$ таковы, что любой разностный оператор из пространства $H(U)$ в $H(D)$, для которого выполнены условия а) и б) теоремы 1 и $s_{r}=c$, имеет замкнутый образ в пространстве $D$.

Тогда сужения функиий $\alpha(x, U)$ и $\beta(x, U)$ на множество $\left(U^{-}, U^{+}\right) \cap$ $\left[D^{-}, D^{+}\right]$будут непрерывнь.

Действительно, из утверждения 3 имеем неравенства

$$
\begin{aligned}
\varliminf_{x \rightarrow x_{0},}, \varlimsup_{x \in\left(D^{-}, D^{+}\right)} \alpha(x, U)>\alpha\left(x_{0}, U\right), \\
\varlimsup_{x \rightarrow x_{0},} \beta\left(x \in\left(D^{-}, D^{+}\right)\right.
\end{aligned}
$$

а обратные неравенства вытекают из полунепрерывности этих функций.

В теореме 3 мы нашли условия на область $U$, при которых замкнутость образа оператора $\mathscr{A}$ эквивалентна замкнутости образа разностного оператора

$$
\mathscr{A}^{\prime}: H(U) \rightarrow H(D),
$$

образованного крайними коэффициентами оператора $\mathscr{A}$.

Покажем сушественность этих условий для подобной связи между операторами $\mathscr{A}$ и $\mathscr{A}^{\prime}$. В нижеследующих примерах число $r$ будет равно двум.

ПРИмеР 1. Пусть

$$
U=\{z \in \mathbb{C}: 0<\operatorname{Im} z<6\} \backslash[0,2 i], \quad D=\{z \in \mathbb{C}: 0<\operatorname{Im} z<4\} \backslash[0,2 i] .
$$

Рассмотрим функции $g_{0}, g_{2} \in H(D)$ такие, что функция $g_{0}$ обрашается в нуль только в точках последовательности $\left\{z_{n} \in D:\left|z_{n}\right|<1, n \in \mathbb{N}\right\}$, все эти нули простые и

$$
\lim _{n \rightarrow \infty} z_{n}=0,
$$

а функция $g_{2}(z)$ обрашается в нуль только в точках $z_{n}+2 i, n \in \mathbb{N}$.

Докажем, что оператор

$$
\left(\mathscr{A}^{\prime} f\right)(z)=g_{0}(z) f(z)+g_{2}(z) f(z+2 i), \quad f \in H(U),
$$

имеет замкнутый образ.

Действительно, пусть функция $G \in H(D)$ принадлежит замыканию образа оператора $\mathscr{A}^{\prime}$, т.е. для некоторой последовательности $f_{m} \in H(U), m \in \mathbb{N}$,

$$
\lim _{m \rightarrow \infty} \mathscr{A} f_{m}=G
$$

в топологии пространства $H(D)$. В таком случае, очевидно,

$$
g_{0}\left(z_{n}+2 i\right) G\left(z_{n}\right)=g_{2}\left(z_{n}\right) G\left(z_{n}+2 i\right), \quad n \in \mathbb{N} .
$$

Обозначим через $U_{1}$ и $D_{1}$ соответственно области

$$
U \backslash\{z \in \mathbb{C}:|z| \leqslant 1\} \text { и } D \backslash\{z \in \mathbb{C}:|z| \leqslant 1\} .
$$


Оператор $\mathscr{A}^{\prime}$, очевидно, отображает пространство $H\left(U_{1}\right)$ в $H\left(D_{1}\right)$ и удовлетворяет условиям теоремы 2 . Так как функция $G(z)$ принадлежит замыканию образа этого оператора, то найдется функция $f \in H\left(U_{1}\right)$, для которой

$$
g_{0}(z) f(z)+g_{2}(z) f(z+2 i)=G(z), \quad z \in D_{1} .
$$

Функции $g_{0}(z), g_{2}(z) f(z+2 i)$ и $G(z)$ голоморфны на множестве

$$
U \cap\{z \in \mathbb{C}:|z| \leqslant 1\}
$$

поэтому и функция $g_{0}(z) f(z)$ будет на нем голоморфна, а для голоморфности функции $f(z)$ на этом множестве достаточно выполнения равенств

$$
G\left(z_{n}\right)=g_{2}\left(z_{n}\right) f\left(z_{n}+2 i\right), \quad n \in \mathbb{N} .
$$

Из равенства (20) получим

$$
g_{0}\left(z_{n}+2 i\right) f\left(z_{n}+2 i\right)=G\left(z_{n}+2 i\right), \quad n \in \mathbb{N},
$$

что вместе с равенствами (19) и неравенствами $g_{2}\left(z_{n}\right) \neq 0, n \in \mathbb{N}$, даст соотношение (21).

Итак, функция $f$ принадлежит $H(U)$, поэтому

$$
\left(\mathscr{A}^{\prime} f\right)(z)=G(z), \quad z \in D
$$

и, следовательно, образ оператора $\mathscr{A}^{\prime}$ замкнут.

Рассмотрим теперь функцию $g_{1} \in H(D), g_{1}\left(z_{n}\right)=0 \neq g_{1}\left(z_{n}+2 i\right), n \in \mathbb{N}$, и докажем, что образ оператора

$$
(\mathscr{A} f)(z)=g_{0}(z) f(z)+g_{1}(z) f(z+i)+g_{2}(z) f(z+2 i), \quad f \in H(U),
$$

не замкнут.

Действительно, для функции $f \in \operatorname{Ker} \mathscr{A}$, как несложно видеть, выполнены равенства

$$
f\left(z_{n}+3 i\right)=0, \quad n \in \mathbb{N} .
$$

Но точка $3 i$ лежит в области $U$, и, следовательно, $f \equiv 0$. Из леммы 4 заключаем, что образ оператора $\mathscr{A}$ не замкнут.

ПримеР 2. Пусть

$$
U=\{z \in \mathbb{C}: 0<\operatorname{Im} z<8\} \backslash[0,2 i], \quad D=\{z \in \mathbb{C}: 0<\operatorname{Im} z<4\} \backslash[0,2 i] .
$$

Рассмотрим функции $g_{0}, g_{2} \in H(D)$ такие, что функция $g_{0}(z)$ обращается в нуль только в точках $\left\{z_{n} \in D:\left|z_{n}\right|<1, n \in \mathbb{N}\right\}$, все эти нули простые и

$$
\lim _{n \rightarrow \infty} z_{n}=0
$$

а функция $g_{2}(z)$ обрашается в нуль только в точках $z_{n}+2 i, n \in \mathbb{N}$.

Положим

$$
g_{1}(z)=g_{0}(z)+g_{2}(z), \quad z \in D
$$


и докажем, что образ оператора

$$
(\mathscr{A} f)(z)=g_{0}(z) f(z)+g_{1}(z) f(z+2 i)+g_{2}(z) f(z+4 i)
$$

замкнут.

Действительно,

$$
(\mathscr{A} f)(z)=g_{0}(z)[f(z)+f(z+2 i)]+g_{2}(z)[f(z+2 i)+f(z+4 i)],
$$

так что оператор $\mathscr{A}$ представляется в виде суперпозищии двух разностных операторов,

$$
\left(\mathscr{A}_{1} f_{1}\right)(z)=f_{1}(z)+f_{1}(z+2 i), \quad f_{1} \in H(U)
$$

$$
\left(\mathscr{A}_{2} f_{2}\right)(z)=g_{0}(z) f_{2}(z)+g_{2}(z) f_{2}(z+2 i), \quad f_{2} \in H(U \cap(U-2 i)) .
$$

Первый оператор, как отмечалось в лемме 5, эпиморфен, а второй, как показано в предыдушем примере, имеет замкнутый образ, поэтому и оператор $\mathscr{A}$ имеет замкнутый образ.

Оператор же

$$
\left(\mathscr{A}^{\prime} f\right)(z)=g_{0}(z) f(z)+g_{2}(z) f(z+4 i), \quad f \in H(U),
$$

имеет незамкнутый образ, ибо, как легко показать, ядро этого оператора нулевое.

Покажем теперь, что аналогичная ситуация имеет место и для разностных операторов в пространстве целых функций.

ПримеР 3. Пусть $U=D=\mathbb{C}$, целые функции $g_{0}$ и $g_{2}$ имеют корни только соответственно в точках $3 n i$ и $(3 n i+2 i), n \in \mathbb{Z}$, и все эти корни простые. Как и в примере 1 , можно показать, что у оператора

$$
\left(\mathscr{A}^{\prime} f\right)(z)=g_{0}(z) f(z)+g_{2}(z) f(z+2 i)
$$

замкнутый образ.

Пусть теперь функция $g_{1} \in H(\mathbb{C})$ удовлетворяет соотношениям

$$
g_{1}(3 n i)=0 \neq g_{1}(3 n i+2 i), \quad n \in \mathbb{N} .
$$

В таком случае оператор

$$
(\mathscr{A} f)(z)=g_{0}(z) f(z)+g_{1}(z) f(z+i)+g_{2}(z) f(z+2 i)
$$

имеет незамкнутый образ.

Действительно, если $f \in \operatorname{Ker} \mathscr{A}$, то несложно показать, что $f(n i)=0, n \in \mathbb{Z}$, и поэтому функция

$$
f_{1}(z)=\frac{f(z)}{e^{2 \pi i z}-1}
$$

также будет лежать в ядре оператора $\mathscr{A}$. Ясно, что такое возможно лишь в случае тождественного равенства нулю функции $f(z)$, и из леммы 4 убеждаемся в незамкнутости образа этого оператора.

ПримеР 4. Пусть $U=D=\mathbb{C}$, целые функции $g_{0}$ и $g_{2}$ имеют корни только соответственно в точках $2 n i$ и $(2 n i+i), n \in \mathbb{Z}$, и все эти корни простые.

Так же, как в примере 2 , можно показать, что оператор

$$
(\mathscr{A} f)(z)=g_{0}(z) f(z)+g_{1}(z) f(z+i)+g_{2}(z) f(z+2 i), \quad f \in H(\mathbb{C}),
$$

где $g_{1}(z)=g_{0}(z)+g_{2}(z)$, имеет замкнутый образ.

Образ же оператора

$$
\left(\mathscr{A}^{\prime} f\right)(z)=g_{0}(z) f(z)+g_{2}(z) f(z+2 i), \quad f \in H(\mathbb{C}),
$$

не замкнут, ибо его ядро нулевое. 


\section{§ 5. Разностные операторы с замкнутым образом}

В данном параграфе мы приведем некоторые свойства разностных операторов с замкнутым образом.

УТВЕРЖДЕНИЕ 4. Пусть образ оператора $\mathscr{A}$ замкнут, область $U_{1} \subset U$, причем множество

$$
D_{1}=U_{1} \cap\left(U_{1}-i s_{r}\right)
$$

связно и для любих точек $x_{1}+i y_{1}, x_{2}+i y_{2} \in D$ таких, что

$$
\begin{gathered}
x_{1}, x_{2} \in\left(U_{1}^{-}, U_{1}^{+}\right), \quad y_{1} \leqslant \alpha\left(x_{1}, U_{1}\right), \quad y_{2}+s_{r} \geqslant \beta\left(x_{2}, U_{1}\right), \\
g_{0}\left(x_{1}+i y_{1}\right)=0=g_{r}\left(x_{2}+i y_{2}\right),
\end{gathered}
$$

выполнены условия:

1) $y_{1}+s_{r} \geqslant \beta\left(x_{1}, U_{1}\right), y_{2} \leqslant \alpha\left(x_{2}, U_{1}\right)$;

2) на множествах

$$
\left\{x_{1}+i y \in D: y \geqslant y_{1}\right\}, \quad\left\{x_{2}+i y \in D: y \leqslant y_{2}\right\}
$$

соответственно функиии $g_{r}(z)$ и $g_{0}(z)$ не обращаются в нуль.

Tогда:

а) если $D_{1}=\varnothing$, то пространство $\operatorname{Ker} \mathscr{A}$ плотно в пространстве $H\left(U_{1}\right)$;

б) если $D_{1} \neq \varnothing$, то пространство $\operatorname{Ker} \mathscr{A}$ плотно в пространстве Ker $\mathscr{A}_{1}$, əде оператор

$$
\mathscr{A}_{1}: H\left(U_{1}\right) \rightarrow H\left(D_{1}\right)
$$

определен по той же формуле, что и оператор $\mathscr{A}$.

ДокАЗАТЕльство. Рассмотрим функционал $T \in H^{*}\left(U_{1}\right)$, аннулирующий пространство $\operatorname{Ker} \mathscr{A}$.

Как указывалось выше, замкнутость образа оператора $\mathscr{A}$ влечет за собой существование функционала $S \in H^{*}(D)$ такого, что

$$
\mathscr{A}^{*} S=T
$$

Обозначим через $\eta(z)$ и $\gamma(z)$ преобразования Коши функционалов $T$ и $S$. Найдется связньй $i$-выпуклый компакт $K \subset U_{1}$, содержащий все особенности функции $\eta(z)$, такой, что компакт $k=K \cap\left(K-i s_{r}\right)$ связен.

По утверждению 1 функция $\gamma(z)$ будет мероморфна вне компакта $k$ с конечным числом полюсов, и согласно следствию этого утверждения можно считать, что вне полосы (5) у этой функции нет особенностей.

Допустим, что для некоторого числа $c \in \mathbb{R}$ на множестве

$$
\{z \in \mathbb{C}: \operatorname{Re} z=c\} \backslash D_{1}
$$

у функции $\gamma(z)$ имеются полюса, $c+i t_{1}-$ самый нижний, а $c+i t_{2}-$ самьй верхний, $t_{1} \leqslant t_{2}$. В таком случае, очевидно, $U_{1}^{-}<c<U_{1}^{+}$.

Один из лучей

$$
\left\{c+i t: t \leqslant t_{1}\right\}, \quad\left\{c+i t: t \geqslant t_{2}+s_{r}\right\}
$$


не будет пересекаться с областью $U_{1}$, иначе, как несложно показать, точки $c+i t_{1}$, $c+i t_{2}$ принадлежат $D_{1}$. Пусть этим свойством обладает первый луч.

Лемма 2 влечет за собой равенство $g_{0}\left(c+i t_{1}\right)=0$, поэтому в силу условия 1$)$ второй луч также не пересекается с областью $U_{1}$, и, значит, $g_{r}\left(c+i t_{2}\right)=0$.

Но это равенство противоречит условию 2), и, следовательно, у функции $\gamma(z)$ не может быть особенностей на множестве $(22)$.

Так как вне компакта $k \subset D_{1}$ у функции $\gamma(z)$ лиш конечное число полюсов, то, выходит, все они лежат на множестве $D_{1}$.

Если $D_{1}=\varnothing$, то у функции $\gamma(z)$ нет особенностей и, следовательно, функционалы $S$ и $T$ нулевые, тогда п. а) вытекает из теоремы Хана-Банаха.

Если же $D_{1} \neq \varnothing$, то $S \in H^{*}\left(D_{1}\right)$, и в силу полноты пространства $H(U)$ в $H\left(U_{1}\right)$ имеем

$$
\mathscr{A}_{1}^{*} S=T \text {. }
$$

В таком случае для любой функции $f \in \operatorname{Ker} \mathscr{A}_{1}$

$$
\langle T, f\rangle=\left\langle\mathscr{A}_{1}^{*} S, f\right\rangle=\left\langle S, \mathscr{A}_{1} f\right\rangle=0,
$$

что и доказывает утверждение.

Покажем теперь точность результата.

ПримеР 5. Для оператора

$$
\begin{gathered}
\mathscr{A}: H(\mathbb{C}) \rightarrow H(\mathbb{C}) \\
(\mathscr{A} f)(z)=\sin \pi i z f(z)+f(z+i)
\end{gathered}
$$

и области

$$
U_{1}=\{z \in \mathbb{C}: \operatorname{Re} z>0\}
$$

выполнены условия утверждения 4 , кроме замкнутости образа оператора $\mathscr{A}$.

Как и в примере 3 , можно показать, что ядро оператора $\mathscr{A}$ нулевое, а по теореме 2 и лемме $4 \operatorname{Ker} \mathscr{A}_{1} \neq\{0\}$, так что полноты не будет.

ПРИМЕР 6. Пусть

$$
\begin{gathered}
\mathscr{A}: H(\mathbb{C}) \rightarrow H(\mathbb{C}), \\
(\mathscr{A} f)(z)=i z f(z)-f(z+i), \\
U_{1}=\{z \in \mathbb{C}: \operatorname{Im} z>0\} .
\end{gathered}
$$

По теореме 2 образ оператора $\mathscr{A}$ замкнут, и, очевидно, выполнены все условия утверждения 4, кроме 1 ).

Здесь имеем

$$
\forall f \in \operatorname{Ker} \mathscr{A} \quad f(i)=0
$$

а в ядре оператора $\mathscr{A}_{1}$ имеется функция

$$
\sin \pi i z \Gamma(-i z)
$$

не имеющая нулей в области $U_{1}$, так что и здесь полноты нет. 
ПримеР 7. Для оператора

$$
\begin{gathered}
\mathscr{A}: H(\mathbb{C}) \rightarrow H(\mathbb{C}), \\
(\mathscr{A} f)(z)=z f(z)+f(z+i)+z f(z+2 i)
\end{gathered}
$$

и области

$$
U_{1}=\{z \in \mathbb{C}: 0<\operatorname{Im} z<2\} \cup\{z \in \mathbb{C}: \operatorname{Re} z>0\}
$$

вьполнены все условия утверждения 4 , кроме 2$)$.

Для любой функции $f \in \operatorname{Ker} \mathscr{A} f(i)=0$, а для пространства $\operatorname{Ker} \mathscr{A}_{1}$, как это следует из теоремы 2 и леммы 3 , это не имеет места.

В случае $D_{1}=\varnothing$ несложно построить аналогичные примеры.

Приведем теперь достаточные условия эпиморфности оператора $\mathscr{A}$.

УТВеРЖДЕНИЕ 5. Пусть образ оператора $\mathscr{A}$ замкнут и для любых точек $z_{1}, z_{2} \in D$ maкux, ито

$$
\operatorname{Re} z_{1}=\operatorname{Re} z_{2}, \quad g_{0}\left(z_{1}\right)=0=g_{r}\left(z_{2}\right),
$$

имеют место неравенства

$$
\operatorname{Re}\left(z_{2}-z_{1}\right)<s_{j+1}-s_{j}, \quad j=0, \ldots, r-1 .
$$

Тогда образ оператора $\mathscr{A}$ совпадает со всем пространством $H(D)$.

ДокАЗАТЕЛЬСТво. Рассмотрим функционал $S \in \operatorname{Ker} \mathscr{A}^{*}$ и обозначим через $\gamma(z)$ его преобразование Коши. Согласно утверждению 1 эта функция будет рациональной.

Допустим, что функция $\gamma(z)$ отлична от тождественного нуля, и представим ее в виде

$$
\gamma(z)=\sum_{m=1}^{n} \gamma_{m}(z)
$$

где функция $\gamma_{m}(z)$ рациональная, ненулевая с единственным полюсом в точке $z_{m} \in$ $D, \gamma_{m}(\infty)=0, m=1, \ldots, n$, и полюсы различных функций не совпадают. Без ограничения обшности можно считать, что все эти полюсы лежат на одной прямой:

$$
z_{m}=c+i y_{m}, \quad m=1, \ldots, n, \quad y_{1}<y_{2}<\cdots<y_{n} .
$$

В таком случае по лемме $2 g_{0}\left(z_{1}\right)=0=g_{r}\left(z_{n}\right)$, и, следовательно, выполнены неравенства

$$
\operatorname{Re}\left(z_{n}-z_{1}\right)<s_{j+1}-s_{j}, \quad j=0, \ldots, r-1 .
$$

По лемме 1 имеем

$$
\sum_{j=0}^{\gamma} \sum_{m=1}^{n} \int_{C} \frac{\gamma_{m}(t) g_{j}(t)}{z-t-i s_{j}} d t=0
$$

где контур $C$ охватывает все полюсы функции $\gamma(z)$, а точка $z$ лежит достаточно далеко от начала координат.

Функция

$$
\int_{C} \frac{\gamma_{m}(t) g_{j}(t)}{z-t-i s_{j}} d t
$$


очевидно, рациональна с единственной особой точкой $\left(z_{m}+i s_{j}\right), m=1, \ldots, n$, $j=0, \ldots, r$. Неравенства (23), как несложно показать, обеспечивают несовпадение этих точек, и, следовательно, все функции (24) равны нулю, что может быть лишь в случае равенств

$$
g_{j}\left(z_{m}\right)=0, \quad m=1, \ldots, n, \quad j=0, \ldots, r .
$$

Но у функций $g_{0}, \ldots, g_{r}$ нет обших нулей, и, значит, функция $\gamma(z)$ тождественно равна нулю.

Итак, ядро оператора $\mathscr{A}^{*}$ нулевое, и искомое следует из замкнутости образа оператора $\mathscr{A}$ (см. [4, с. 705]).

Покажем теперь, что условие на нули в этом результате нельзя ослабить.

ПримеР 8. Рассмотрим оператор

$$
\begin{gathered}
\mathscr{A}: H(\mathbb{C}) \rightarrow H(\mathbb{C}) \\
(\mathscr{A} f)(z)=z f(z)+(z-i) f(z+i) .
\end{gathered}
$$

По теореме 2 оператор $\mathscr{A}$ имеет замкнутый образ и для любой функции $F$ из этого образа, очевидно, выполнено равенство

$$
F(0)+F(i)=0
$$

так что

$$
\mathscr{A}(H(\mathbb{C})) \neq H(\mathbb{C})
$$

\section{Список литературы}

1. Мерзляков С.Г. О замкнутости образа разностного оператора // ДАН. 1993. Т. 328. № 2. C. 141-142.

2. Köte G. Dualität in der Functionentheorie // J. reine und angew. Math. 1953. B. 191. № 1-2. S. 30-49.

3. Мерзляков С.Г. Инвариантные подпространства оператора единичного сдвига // Изв. РАН. Сер. матем. 1992. Т. 56. №6. С. 1244-1272.

4. Эдвардс Р. Функциональный анализ. М.: Мир, 1969.

5. Рудин У. Функциональный анализ. М.: Мир, 1975.

6. Мерзляков С.Г. О возмущении линейных операторов в пространствах голоморфных функций // Матем. сб. 1995. Т. 186. № 3. С. 103-130.

7. Себастьян- $и$-Сильва Ж. О некоторых классах локально выпуклых пространств, важных в приложениях // Математика. 1957. Т. 1. № 1. С. 60-77.

Поступило в редакцию 29.VIII.1995 